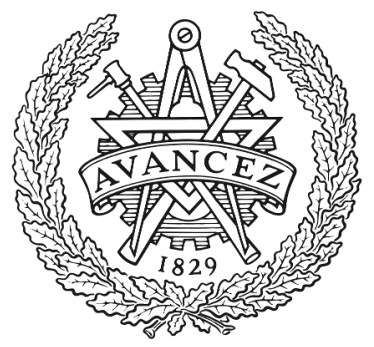

CHALMERS

UNIVERSITY OF TECHNOLOGY

\title{
5G mmWave Vehicular Tracking
}

Downloaded from: https://research.chalmers.se, 2023-04-26 05:15 UTC

Citation for the original published paper (version of record):

Kim, H., Wymeersch, H., Garcia, N. et al (2019). 5G mmWave Vehicular Tracking. Conference Record - Asilomar Conference on Signals, Systems and Computers, 2018-October: 541-547. http://dx.doi.org/10.1109/ACSSC.2018.8645553

N.B. When citing this work, cite the original published paper. 


\title{
5G mmWave Vehicular Tracking
}

\author{
Hyowon Kim*, Henk Wymeersch ${ }^{\dagger}$, Nil Garcia ${ }^{\dagger}$, Gonzalo Seco-Granados ${ }^{\ddagger}$, Sunwoo Kim* \\ $\dagger$ Department of Electrical Engineering, Chalmers University of Technology, Sweden \\ * Department of Electronic Engineering, Hanyang University, Seoul, Korea \\ $\ddagger$ Department of Telecommunications and Systems Engineering, Universitat Autonoma de Barcelona, Spain
}

\begin{abstract}
Vehicle positioning based on GPS is limited due to multipath and blockage. 5G mmWave signals can provide an attractive complement, as it is possible to estimate the state of a vehicle (position and heading) from transmissions from a single base station. We propose a Bayesian $5 \mathrm{G}$ mmWave tracking filter, which explicitly relies on mapping the radio environment. The filter thus solves a novel type of simultaneous localization and mapping problem, which enables estimating not only the vehicle heading and position, but also its clock bias.
\end{abstract}

\section{INTRODUCTION}

Vehicles can rely on a combination of GPS (for absolute positioning), radar, lidar, and stereo camera (for relative positioning) to determine their position in the environment. An important difference is in how these systems deal with multipath (i.e., signals reflected or scattered in the environment): in GPS multipath is considered a nuisance [1], which should be suppressed or mitigated. In contrast, multipath is what brings useful information in radar and lidar, in the forms of distances, velocities, or angles [2]. Recently, 5G mmWave has been proposed as a way to localize vehicles from signals from a fixed reference (a base station (BS)), as well as to map the environment by harnessing multipath, and subsequently to exploit this map to improve the absolute localization [3]. An important benefit of $5 \mathrm{G}$ localization is that it can use existing communication hardware and thus provide a low-cost complement to existing solutions.

There is an extensive body of work (both theoretical and experimental) exploiting multipath for simultaneous localization and mapping (SLAM) based on time of arrival (TOA) measurements [4]. The SLAM problem can be solved using a variety of techniques, including a Rao-Blackwellized particle filter with external data association [5], and using a factor graph with internal data association [6]. More recently, the use of 5G mmWave was considered for similar problems [7]. The properties of $5 \mathrm{G}$ mmWave signals are particularly amenable for positioning and mapping, as they provide information regarding not only TOA, but also direction of arrival (DOA) and direction of departure (DOD) [8]. In [9], it was shown that these measurements allow SLAM based on a snapshot measurement (i.e., without prior information), even when transmitter and receiver are not synchronized. The work in [9] was however limited to an environment with only reflecting surfaces.

In this paper, we extend [9] by considering both reflecting surfaces and scattering points (SP). We show how each path provides useful location information, even when its source is

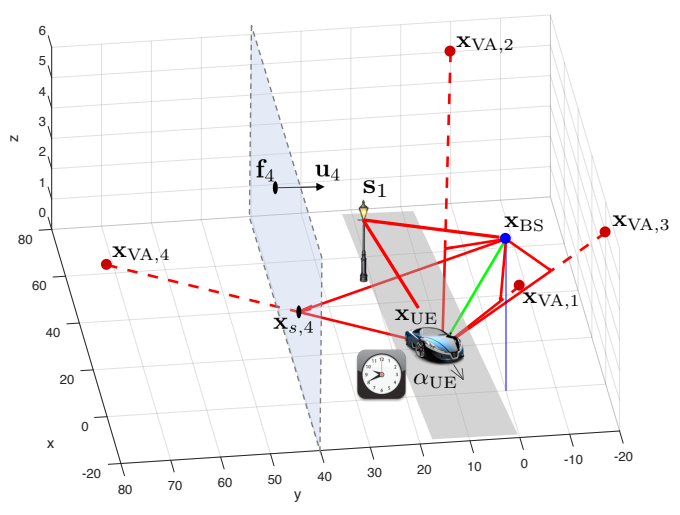

Fig. 1. Scenario with one base station (blue), one vehicle (heading shown with an arrow), a scattering point, and 4 virtual anchors (each corresponding to a vertical wall, one of which is shown).

a priori unknown. Moreover, we provide a method that is able to disambiguate between virtual anchors (VA) and scattering points. Our method combines three main components: (i) a hard data association routine to match paths to map entries; (ii) a belief propagation filter to compute posterior distributions over the vehicle state and map entries; (iii) a routine to add and remove entries in the map, including a way to distinguish between different types of sources.

\section{System Model And Problem Formulation}

\section{A. State Model}

We consider a scenario as shown in Fig. 1 with a single static base station (BS) and a single mobile user equipment (UE) mounted on a vehicle. The BS is located at $\mathbf{x}_{\mathrm{BS}}=$ $\left[0,0, z_{\mathrm{BS}}\right]^{\mathrm{T}} \in \mathbb{R}^{3}$, while the UE has a 7-dimensional state $\mathbf{s}_{k}=\left[\mathbf{x}_{k}^{\mathrm{T}}, \alpha_{k}, v_{k}, \omega_{k}, B_{k}\right]^{\mathrm{T}}$, comprising the vehicle position and orientation, as well as longitudinal and rotational velocity and clock bias. The vehicle dynamics follow a constant turn rate velocity model [10, Chapter 5], [11]:

$$
\begin{aligned}
& \mathbf{s}_{k}=\mathbf{s}_{k-1}+ \\
& {\left[\begin{array}{c}
\frac{v_{k-1}}{\omega_{k-1}}\left(\sin \left(\alpha_{k-1}+\omega_{k-1} \Delta\right)-\sin \alpha_{k-1}\right) \\
\frac{v_{k-1}}{\omega_{k-1}}\left(-\cos \left(\alpha_{k-1}+\omega_{k-1} \Delta\right)+\cos \alpha_{k-1}\right) \\
0 \\
\omega_{k-1} \Delta \\
\mathbf{0}_{3}
\end{array}\right]+\mathbf{w}_{k},}
\end{aligned}
$$

where $\mathbf{0}_{m}$ denotes a column vector of $m$ zeros, $\Delta$ is the duration of an epoch and $\mathbf{w}_{k}$ denotes the process noise, 
modeled as zero-mean Gaussian with covariance $\mathbf{Q}=$ $\operatorname{diag}\left[\mathbf{0}_{4}^{\mathrm{T}}, \sigma_{v}^{2}, \sigma_{\omega}^{2}, \sigma_{B}^{2}\right]$.

The BS and UE communicate with each other in an environment characterized by reflecting surfaces and SPs (corresponding to small objects). The SPs have fixed locations $\mathbf{x}_{\mathrm{SP}, m} \in \mathbb{R}^{3}$, while each reflecting surface can be parameterized by a fixed virtual anchor location $\mathbf{x}_{\mathrm{VA}, m}$, obtained by mirroring ${ }^{1}$ the $\mathrm{BS}$ with respect to the surface. With each reflecting surface, we can also associate a moving incidence point, defined as the intersection of the line between the VA and UE position with the surface.

\section{B. Measurement Model}

The BS periodically sends a mmWave positioning reference signal (PRS). At epoch $k$, the received signal at the UE depends on the mmWave propagation channel [12], of the form $\mathbf{H}_{k}(t)=\sum_{l=0}^{L_{k}-1} h_{l, k} \mathbf{a}_{\mathrm{UE}}\left(\boldsymbol{\theta}_{l, k}\right) \mathbf{a}_{\mathrm{BS}}^{\mathrm{H}}\left(\phi_{l, k}\right) \delta\left(t-\tau_{l, k}\right)$, where $L_{k}$ is the number of resolvable propagation paths, $h_{l, k}$ is a complex channel gain, $\mathbf{a}_{\mathrm{UE}}(\cdot)$ and $\mathbf{a}_{\mathrm{BS}}(\cdot)$ are the antenna response vectors, and $\tau_{l, k}, \boldsymbol{\theta}_{l, k}$, and $\boldsymbol{\phi}_{l, k}$ denote the TOA, DOA and DOD, respectively. Both DOA and DOD have azimuth and elevation components. Each of the $L_{k}$ paths comes from either the line-of-sight (LOS) link, or is a multipath component due to a SP or a reflecting surface. The relations between the channel parameters and the location parameters are detailed in the Appendix.

We further assume that the UE employs a channel estimation method to recover the triplet of TOA, DOA, and DOD, e.g., based on sparse recovery [8] or subspace methods [13], which achieve a good balance between estimation accuracy and computational complexity. A channel estimator thus provides

$$
\mathbf{z}_{l, k}=\left[\tau_{l, k}, \boldsymbol{\theta}_{l, k}^{\mathrm{T}}, \boldsymbol{\phi}_{l, k}^{\mathrm{T}}\right]^{\mathrm{T}}+\mathbf{n}_{l, k},
$$

where $\mathbf{n}_{l, k} \sim \mathcal{N}\left(\mathbf{0}, \boldsymbol{\Sigma}_{l, k}\right)$, in which $\boldsymbol{\Sigma}_{l, k}$ depends on the channel as well as any precoding, combining, duration of the training signal, etc. Let the measurement set be $\mathbf{Z}_{k}=$ $\left\{\mathbf{z}_{l, k}\right\}_{l=0}^{L-1}$. Note that this set has no ordering.

\section{Problem Formulation}

Given (possibly uninformative) priori information $p\left(\mathbf{s}_{0}\right)$ and in the absence of knowledge regarding the SPs or VAs, our goal is to track the vehicle state (i.e., compute $p\left(\mathbf{s}_{k} \mid \mathbf{Z}_{1: k}\right)$ ) and simultaneously map the environment (i.e., discover SPs and VAs, and compute $p\left(\mathbf{x}_{\mathrm{SP}, m} \mid \mathbf{Z}_{1: k}\right)$ and $\left.p\left(\mathbf{x}_{\mathrm{VA}, m} \mid \mathbf{Z}_{1: k}\right)\right)$.

\section{Methodology}

In this section, we outline the proposed solution, including how we encode the map, how we perform data association, and how the map is updated.

\footnotetext{
${ }^{1}$ Mathematically, the reflecting surface can be described by a point $\mathbf{f}_{m}$ and a normal vector $\mathbf{u}_{m}$. With each reflecting surface we can associate a virtual anchor location $\mathbf{x}_{\mathrm{VA}, m}=\mathbf{P}_{m} \mathbf{x}_{\mathrm{BS}}+\mathbf{t}_{m}$, where $\mathbf{P}_{m}=\mathbf{I}_{3}-2 \mathbf{u}_{m} \mathbf{u}_{m}^{\mathrm{T}}$ is a Householder matrix and $\mathbf{t}_{m}=2 \mathbf{f}_{m}^{\mathrm{T}} \mathbf{u}_{m} \mathbf{u}_{m}$ is a translation vector.
}

\section{A. Belief Structure}

1) UE State: We maintain a belief of the UE state $p\left(\mathbf{s}_{k} \mid \mathbf{Z}_{1: k}\right)$ at the end of epoch $k$. This belief can be in parametric form (e.g, a 7D Gaussian) or in non-parametric form (e.g., a list of particles). We also compute a predicted belief $p\left(\mathbf{s}_{k+1} \mid \mathbf{Z}_{1: k}\right)$, based on the process model (1).

2) Map State: We maintain a belief of the map state. The time-varying map $\mathcal{M}_{k}$ comprises the previously seen signal sources. These include the BS, as well as previously seen VAs, and SPs. In addition, the map also contains unconfirmed sources (e.g., sources for which we are not yet sure if they are VA or SP, or clutter). Hence, for each source, indexed by $m$ we maintain the type $T_{m, k} \in\{\mathrm{BS}, \mathrm{VA}, \mathrm{SP}\}$ as well as a spatial probability density function (pdf) $p_{k}\left(\mathbf{x}_{m} \mid T_{m, k}\right)$ of source $m$ at time $k .^{2}$ The belief is then the aggregation of the beliefs of the individual map entries. For confirmed sources, we have that $\mathrm{P}\left(T_{m, k}\right) \in\{0,1\}$, while for unconfirmed sources $\mathrm{P}\left(T_{m, k}\right) \in(0,1)$,

3) Initialization: At time $k=0$, the UE belief is $p\left(\mathbf{s}_{0}\right)$ and the map is initialized $\mathcal{M}_{0}$ as having a single entry with $\mathrm{P}\left(T_{1,0}=\mathrm{BS}\right)=1$ and $p_{0}\left(\mathbf{x}_{1} \mid T_{1,0}=\mathrm{BS}\right)=\delta\left(\mathbf{x}_{1}-\mathbf{x}_{\mathrm{BS}}\right)$, corresponding to the known BS location.

\section{B. Data Association}

At the beginning of epoch $k$, we are provided with the predicted UE belief $p\left(\mathbf{s}_{k}\right) \doteq p\left(\mathbf{s}_{k} \mid \mathbf{Z}_{1: k-1}\right)$ as well as the map $\mathcal{M}_{k-1}$, with $\left|\mathcal{M}_{k-1}\right| \geq 1$ entries. From the channel estimation routine, we obtain $\mathbf{Z}_{k}$, comprising $L_{k}$ 5-tuples. We define a $L_{k} \times\left(L_{k}+\left|\mathcal{M}_{k-1}\right|\right)$ matrix $\mathbf{S}$, where the rows corresponds to the measurements and the columns to the possible sources of the measurements. We add $L$ columns, since each measurement could have come from a new source that had not previously seen, leading to $\mathbf{S}=\left[\mathbf{S}^{\mathrm{D}} P_{\mathrm{N}} \mathbf{I}_{L_{k}}\right]$, where $\mathbf{I}_{L_{k}}$ is an $L_{k} \times L_{k}$ identity matrix, $P_{\mathrm{N}}$ is the new target rate, and $\mathbf{S}^{\mathrm{D}}$ is an $L_{k} \times\left|\mathcal{M}_{k-1}\right|$ matrix with entries

$$
S_{l, m}^{\mathrm{D}}=\frac{P_{\mathrm{D}}}{1-P_{\mathrm{D}}} p\left(\mathbf{z}_{l, k} \mid b_{l, k}=m\right)
$$

in which $P_{\mathrm{D}}$ is the detection probability and $b_{l, k} \in$ $\left\{1, \ldots,\left|\mathcal{M}_{k-1}\right|\right\}$ represents the source of measurement $\mathbf{z}_{l, k}$. We note that

$$
\begin{aligned}
& p\left(\mathbf{z}_{l, k} \mid b_{l, k}=m\right)= \\
& \sum_{t \in\{\mathrm{BS}, \mathrm{SP}, \mathrm{VA}\}} p\left(T_{m, k-1}=t\right) p\left(\mathbf{z}_{l, k} \mid b_{l, k}=m, T_{m, k-1}=t\right)
\end{aligned}
$$

where, for $t \in\{\mathrm{BS}, \mathrm{SP}, \mathrm{VA}\}$

$$
\begin{aligned}
& p\left(\mathbf{z}_{l, k} \mid b_{l, k}=m, T_{m, k-1}=t\right)= \\
& \int p\left(\mathbf{s}_{k}\right) p_{m, k}\left(\mathbf{x}_{m} \mid t\right) p\left(\mathbf{z}_{l, k} \mid \mathbf{s}_{k}, \mathbf{x}_{m}, t\right) \mathbf{d s}_{k} \mathrm{~d} \mathbf{x}_{m}
\end{aligned}
$$

\footnotetext{
${ }^{2}$ Note that $\mathbf{x}_{m}$ refers to the location of the $m$-th source in the map, while $\mathbf{x}_{k}$ is the UE position at time $k$.
} 
The likelihood function is always of the form

$$
\begin{aligned}
& p\left(\mathbf{z}_{l, k} \mid \mathbf{s}_{k}, \mathbf{x}_{m}, t\right)=\frac{1}{\sqrt{(2 \pi)^{5}\left|\mathbf{\Sigma}_{l, k}\right|}} \times \\
& \exp \left(-\frac{1}{2}\left(\mathbf{z}_{l, k}-\boldsymbol{\eta}\left(\mathbf{s}_{k}, \mathbf{x}_{m}, t\right)\right)^{\mathrm{T}} \boldsymbol{\Sigma}_{l, k}^{-1}\left(\mathbf{z}_{l, k}-\boldsymbol{\eta}\left(\mathbf{s}_{k}, \mathbf{x}_{m}, t\right)\right)\right),
\end{aligned}
$$

where $|$.$| is the determinant, \boldsymbol{\Sigma}_{l, k}$ was defined in (2), and the function $\boldsymbol{\eta}\left(\mathbf{s}_{k}, \mathbf{x}_{m}, t\right)$ comprises the TOA, DOA, and DOD computed according to the Appendix from the location and type $t$ of the $m$-th source and the UE state. The integrals can be readily evaluated using Monte Carlo integration. We note that for confirmed sources, only one term in the sum (4) remains, while for unconfirmed sources, we sum over all possible hypotheses.

In this paper, we consider a simple technique based on the global nearest neighbor assignment [14], which provides hard decisions regarding the associations of measurements to sources. Given the matrix $\mathbf{S}$, we find an optimal assignment by solving the following optimization problem:

$$
\begin{aligned}
\operatorname{maximize} & \sum_{l=0}^{L_{k}-1} \sum_{m=1}^{\left|\mathcal{M}_{k-1}\right|+L_{k}} a_{l, m} \log S_{l, m} \\
\text { s.t. } & a_{l, m} \in\{0,1\} \forall l, m, \\
& \sum_{m=1}^{\left|\mathcal{M}_{k-1}\right|+L_{k}} a_{l, m}=1, \forall l, \\
& \sum_{l=0}^{L_{k}-1} a_{l, m} \leq 1, \forall m,
\end{aligned}
$$

which can be solved efficiently with the Kuhn-Munkres algorithm [15], [16]. This approach can thus determine which measurements come from which source (BS, VA, SP) or from a previously seen unconfirmed source, or from a new source. In particular, when $a_{l, m}=1$ for some $m>\left|\mathcal{M}_{k-1}\right|$, a new source with index $m=\left|\mathcal{M}_{k-1}\right|+1$ is added to the map with $\mathrm{P}\left(T_{m, k}=\mathrm{BS}\right)=0, \mathrm{P}\left(T_{m, k}=\mathrm{VA}\right)=\mathrm{P}\left(T_{m, k}=\mathrm{SP}\right)=1 / 2$ and undefined spatial pdf. Finally, we set the predicted map $\mathcal{M}_{k}$ to $\mathcal{M}_{k-1}\left(\right.$ and $p\left(\mathcal{M}_{k}\right)$ to $p\left(\mathcal{M}_{k-1}\right)$ ).

Remark 1 (Clutter). The proposed approach works under the condition that (i) sources are sufficiently well separated; (ii) there is no clutter. As we are operating in the mmWave regime, spatial separation is plausible [17]. In terms of clutter, we consider that clutter is itself not harmful, provided it has a physical meaning/origin. In that case, the measurements $\mathbf{z}_{l, k}$ have a physical interpretation and can thus be exploited during positioning. The nature of clutter is that it may appear intermittent and thus will not become part of the confirmed map entries.

\section{UE and Map Belief Update}

Given the association at the current epoch, we execute message passing on a factor graph. In this factor graph, we use all measurements (coming from both confirmed and unconfirmed sources) and factorize the full posterior of $p\left(\mathbf{s}_{k}, \mathcal{M}_{k} \mid \mathbf{Z}_{1: k}\right)$ as

$$
\begin{aligned}
& p\left(\mathbf{s}_{k}, \mathcal{M}_{k} \mid \mathbf{Z}_{1: k}\right)=p\left(\mathbf{s}_{k} \mid \mathbf{Z}_{1: k-1}\right) \prod_{l=0}^{L_{k}-1} p\left(\mathbf{z}_{l, k} \mid \mathbf{s}_{k}, \mathbf{x}_{m(l)}, T_{m(l), k}\right) \\
& \times \prod_{m=1}^{\left|\mathcal{M}_{k}\right|} \mathrm{P}\left(T_{m, k} \mid \mathbf{Z}_{1: k}\right) p_{k-1}\left(\mathbf{x}_{m} \mid T_{m, k}, \mathbf{Z}_{1: k}\right)
\end{aligned}
$$

where $m(l)$ is the unique value of $m$ for which $a_{l, m}=$ 1. After message passing, we thus obtain $p\left(\mathbf{s}_{k} \mid \mathbf{Z}_{1: k}\right)$ and $p\left(\mathcal{M}_{k} \mid \mathbf{Z}_{1: k}\right)$, where the latter comprises $p\left(\mathbf{x}_{m} \mid T_{m, k}, \mathbf{Z}_{1: k}\right)$ and $\mathrm{P}\left(T_{m, k} \mid \mathbf{Z}_{1: k}\right)$ for all entries in the map (including unconfirmed sources). The details of message passing are explained in Section IV.

\section{Map Maintenance}

After a number of time steps, $\mathrm{P}\left(T_{m, k} \mid \mathbf{Z}_{1: k}\right)$ will tend to a degenerate distribution, since for a real VA, the hypothesized SP will move along the surface and will thus not be a fixed point. Similarly, for a real SP, the hypothesized VA location will move and thus also not be a fixed point. Hence, when $\log \left(\mathrm{P}\left(T_{m, k}=\mathrm{VA} \mid \mathbf{Z}_{1: k}\right) / \mathrm{P}\left(T_{m, k}=\mathrm{SP} \mid \mathbf{Z}_{1: k}\right)\right)$ is a very large positive (or negative) number, the source is promoted to a VA (or an SP) and considered as confirmed. Note that the distinction between confirmed an unconfirmed is not strictly needed, but helps computationally. Sources that have not been seen for some time, or that only appear in few maps can be discarded according to a forgetting rule. Finally, we also check if two sources are spatially close, in which case they are merged and the map size reduced.

\section{Vehicular Tracking With Message PAssing}

\section{A. Complexity Reduction}

For computational reasons, it is useful to ignore information from unconfirmed sources as they lead to uninformative messages. We can achieve this as follows: for each unconfirmed source, we ignore any prior information from previous epochs during message passing and only compute the location of the incidence point, denoted as $\mathbf{x}_{s, m}$. We thus compute $p\left(\mathbf{x}_{\mathrm{SP}, m} \mid \mathbf{z}_{k}\right)=p\left(\mathbf{x}_{s, m} \mid \mathbf{z}_{k}\right)$ and (in combination with $p\left(\mathbf{s}_{k} \mid \mathbf{Z}_{1: k}\right)$ ) we also determine a hypothesized VA density $p\left(\mathbf{x}_{\mathrm{VA}, m} \mid \mathbf{z}_{k}\right)$. By evaluating the evolution of $p\left(\mathbf{x}_{\mathrm{SP}, m} \mid \mathbf{z}_{k}\right)$ and $p\left(\mathbf{x}_{\mathrm{VA}, m} \mid \mathbf{z}_{k}\right)$ as a function of $k$, we can, after some time, make a hard decision on whether the source was a SP or a VA. ${ }^{3}$ This procedure alleviates the need to track the $T_{m, k}$ variables and reduces overall complexity.

\footnotetext{
${ }^{3}$ In our implementation, we evaluated the difference between the mean of $p\left(\mathbf{x}_{\mathrm{SP}, m} \mid \mathbf{z}_{k_{m}}\right)$ and of $p\left(\mathbf{x}_{\mathrm{SP}, m} \mid \mathbf{z}_{k}\right)$, where $k$ is the current time and $k_{m}$ the first time we saw source $m$. This difference is weighed by the covariance of $p\left(\mathbf{x}_{\mathrm{SP}, m} \mid \mathbf{z}_{k}\right)$ and compared against the same metric for $\mathbf{x}_{\mathrm{VA}, m}$. When one value for the one hypothesis (e.g., SP) is significantly larger than the value for the other hypothesis (i.e., VA), the second hypothesis is confirmed.
} 


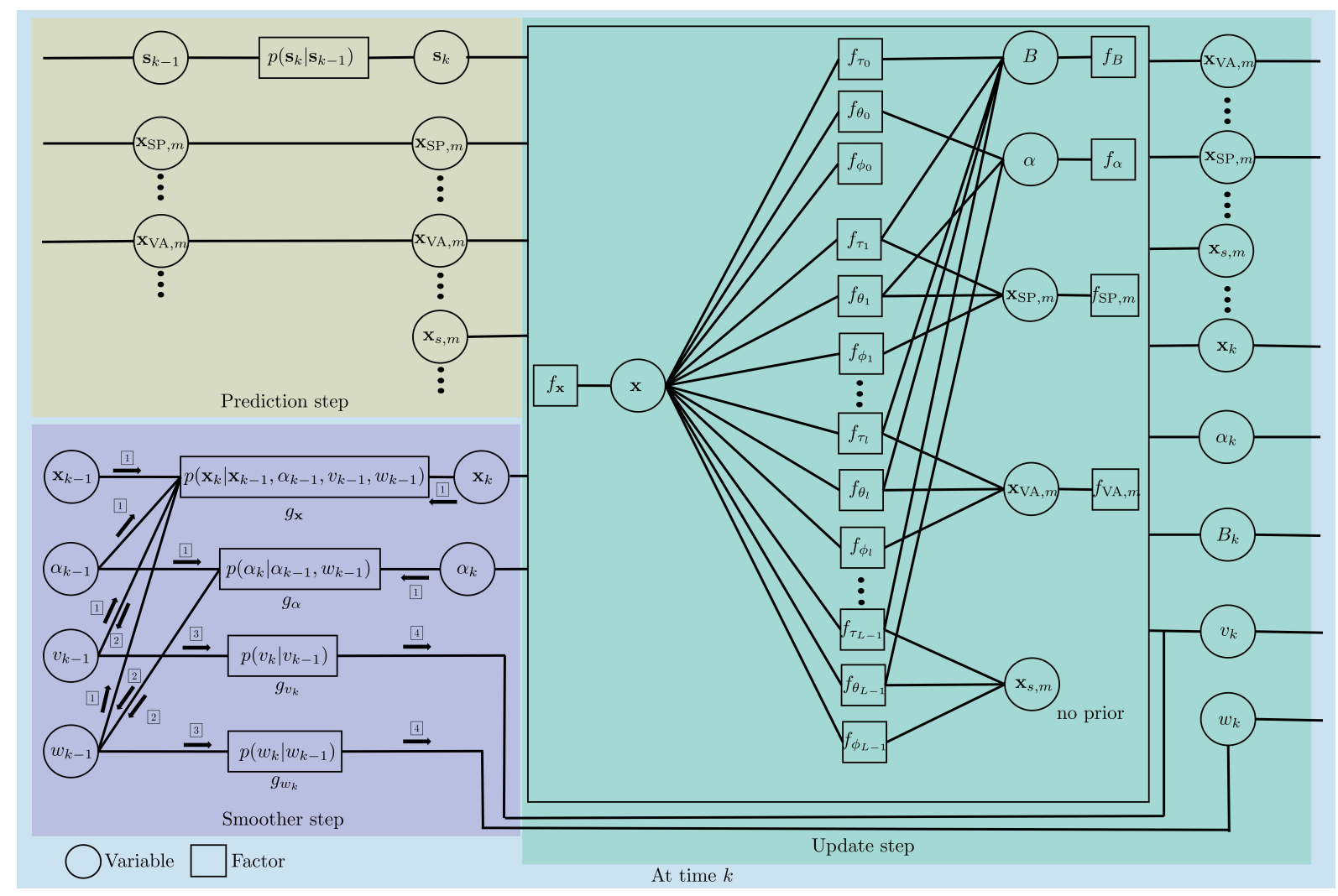

Fig. 2. Factor graph representation of (8) and the message passing schedule. Here, $f_{X}$ refers either to the prior of state variable $X$ or the likelihood of the estimate of a channel parameter $X$.

\section{B. Factor Graph}

The factor graph related to the factorization (8) (with the removal of $T_{m, k}$ as described in Section IV-A) is depicted in Fig. 2. The factor graph includes the individual measurements and their components through likelihood factors. The factor graph assumes that the data association has been completed and thus has three parts: (i) a prediction step (top left part of the figure), where the UE state at the current time is predicted; (ii) an update step (right part of the figure) based on the measurements and a priori information of the UE state as well as from confirmed sources using loopy belief propagation; (iii) a smoothing step (bottom left part of the figure) to account for the measurements in the estimation of the longitudinal and rotational velocity. This is done because the UE does not receive any measurement related to $v_{k}$ and $w_{k}$, so that the marginal posteriors $p\left(v_{k} \mid \mathbf{Z}_{1: k}\right)$ and $p\left(w_{k} \mid \mathbf{Z}_{1: k}\right)$ cannot be directly determined, but can be indirectly obtained via the 1step smoother.

\section{Prediction Step}

Given a prior $p\left(\mathbf{s}_{k-1} \mid \mathbf{Z}_{1: k-1}\right)$ we use the model (1) to predict the UE state as $p\left(\mathbf{s}_{k} \mid \mathbf{Z}_{1: k-1}\right) \propto$ $\int p\left(\mathbf{s}_{k-1} \mid \mathbf{Z}_{1: k-1}\right) p\left(\mathbf{s}_{k} \mid \mathbf{s}_{k-1}\right) \mathrm{d} \mathbf{s}_{k-1}$. The prediction of the sources in the map is done as described at the end of Section III-B. We will denote the map priors of the confirmed sources as $p_{k}\left(\mathbf{x}_{\mathrm{VA}, m}\right)$ and $p_{k}\left(\mathbf{x}_{\mathrm{SP}, m}\right)$.

\section{Update Step}

We now perform iterative message passing to compute beliefs of these five UE state variables: $\mathbf{x}, B, \alpha$, and the beliefs of the confirmed sources $\mathbf{x}_{\mathrm{VA}, m}, \mathbf{x}_{\mathrm{SP}, m}$ for which the data association has assigned a measurement, and incidence points $\mathbf{x}_{s, m}$ of unconfirmed sources. We drop the time index $k$ for notational reasons.

(0) The priors $p(\mathbf{x}), p(B), p(\alpha), p\left(\mathbf{x}_{\mathrm{VA}, m}\right)$, and $p\left(\mathbf{x}_{\mathrm{SP}, m}\right)$ are sent to linked variables. At epoch $k=1$, the LOS DOD message and prior of UE position are combined into a message $\mu_{\mathbf{x}}(\mathbf{x})=\mu_{f_{\mathbf{x}} \rightarrow \mathbf{x}}(\mathbf{x}) \mu_{f_{\phi_{0}} \rightarrow \mathbf{x}}(\mathbf{x})$ since the LOS DOD factor is linked to one variable, LOS DOD message $\mu_{f_{\phi_{0}} \rightarrow \mathbf{x}}(\mathbf{x})$ is informative. Otherwise, LOS DOD message is utilized at step (4).

(1) The message $\mu_{\mathbf{x}}(\mathbf{x})$ is sent to all linked TOA, DOA, DOD likelihoods for all paths (except DOD for LOS path), and the messages $\mu_{\mathbf{x}_{\mathrm{VA}, m}}\left(\mathbf{x}_{\mathrm{VA}, m}\right)$ and $\mu_{\mathbf{x}_{\mathrm{SP}, m}}\left(\mathbf{x}_{\mathrm{SP}, m}\right)$ are sent to linked TOA, DOA, DOD likelihoods.

(2) Except for LOS DOD, each likelihood sends a message to bias or orientation. For instance, the message from the TOA likelihood $f_{\tau_{l}}\left(\mathbf{x}, B, \mathbf{x}_{\mathrm{VA}, m}\right)$ to $B$ is given by

$$
\begin{aligned}
\mu_{f_{\tau_{l}} \rightarrow B}(B) & =\int \mu_{\mathbf{x}}(\mathbf{x}) \mu_{\mathbf{x}_{\mathrm{VA}, m} \rightarrow f_{\tau_{l}}}\left(\mathbf{x}_{\mathrm{VA}, m}\right) \\
& \times f_{\tau_{l}}\left(\mathbf{x}, B, \mathbf{x}_{\mathrm{VA}, m}\right) \mathrm{d} \sim\{B\},
\end{aligned}
$$


where $\mathrm{d} \sim\{B\}$ denotes integration over all variables except $B$.

(3) Now the bias and orientation have been updated, they can send a message back to the likelihood functions. For instance, the message from $B$ to $f_{\tau_{l}}\left(\mathbf{x}, B, \mathbf{x}_{\mathrm{VA}, m}\right)$ is given by $\mu_{B \rightarrow f_{\tau_{l}}}(B)=p(B) \prod_{l^{\prime} \neq l} \mu_{f_{\tau_{l^{\prime}}} \rightarrow B}(B)$.

(4) All likelihoods (except for LOS DOD at epoch $k=$ 1) send messages to the linked variables ( $\mathbf{x}, \mathbf{x}_{\mathrm{VA}, m}$, $\mathbf{x}_{\mathrm{SP}, m}$, and $\mathbf{x}_{\mathrm{s}, m}$ ). For instance, the message from $f_{\tau_{l}}\left(\mathbf{x}, B, \mathbf{x}_{\mathrm{VA}, m}\right)$ to $\mathbf{x}$ is given by

$$
\begin{aligned}
& \mu_{f_{\tau_{l} \rightarrow \mathbf{x}}}(\mathbf{x})=\int \mu_{B \rightarrow f_{\tau_{l}}}(B) \\
& \times \mu_{\mathbf{x} \mathrm{VA}, m} \rightarrow f_{\tau_{l}}\left(\mathbf{x}_{\mathrm{VA}, m}\right) f_{\tau_{l}}\left(\mathbf{x}, B, \mathbf{x}_{\mathrm{VA}, m}\right) \mathrm{d} \sim\{\mathbf{x}\} .
\end{aligned}
$$

Now, the outgoing messages from the position of UE, VA, and SP towards all the likelihood functions can be computed, so we can go back to step (1).

After a sufficient number of iterations between steps (1)(4), the message passing in the update step is stopped and approximate marginal posteriors are found by multiplication of all incoming messages to the associated variables. For instance, for the UE position,

$$
p\left(\mathbf{x}_{k} \mid \mathbf{Z}_{1: k}\right) \propto p(\mathbf{x}) \prod_{l=0}^{L-1} \mu_{f_{\tau_{l} \rightarrow \mathbf{x}}}(\mathbf{x}) \mu_{f_{\theta_{l}} \rightarrow \mathbf{x}}(\mathbf{x}) \mu_{f_{\phi_{l}} \rightarrow \mathbf{x}}(\mathbf{x}),
$$

and similarly for all the other variables. Given $p_{k}\left(\mathbf{x}_{s, m}\right)$ and $p\left(\mathbf{x}_{k} \mid \mathbf{Z}_{1: k}\right)$, then $p_{k}\left(\mathbf{x}_{\mathrm{VA}, m}\right)$ can be calculated from (12).

\section{E. 1-Step Smoother}

The message passing along with scheduling in the 1-step smoother is performed as follows.

1 The messages from $\mathbf{x}_{k}$ and $\alpha_{k}$ to linked likelihoods are computed as $\lambda_{\mathbf{x}_{k} \rightarrow g_{\mathbf{x}}}\left(\mathbf{x}_{k}\right)=p\left(\mathbf{x}_{k} \mid \mathbf{Z}_{1: k}\right) / p\left(\mathbf{x}_{k}\right)$ and $\lambda_{\alpha_{k} \rightarrow g_{\alpha}}\left(\alpha_{k}\right)=p\left(\alpha_{k} \mid \mathbf{Z}_{1: k}\right) / p\left(\alpha_{k}\right)$. The messages $\mathbf{x}_{k-1}$, $\alpha_{k-1}, v_{k-1}$, and $w_{k-1}$ to linked likelihoods are the prior at epoch $k-1$.

2 The likelihood $g_{\mathbf{x}}$ and $g_{\alpha}$ send messages to linked variables. For instance, the message $\lambda_{g_{\mathbf{x}} \rightarrow w_{k-1}}\left(w_{k-1}\right)$ is given by

$$
\begin{aligned}
& \lambda_{g_{\mathbf{x}} \rightarrow w_{k-1}}\left(w_{k-1}\right)=\int p\left(\mathbf{x}_{k} \mid \mathbf{x}_{k-1}, \alpha_{k-1}, v_{k-1}, w_{k-1}\right) \\
& \times p\left(\mathbf{x}_{k-1}\right) p\left(\alpha_{k-1}\right) p\left(v_{k-1}\right) \lambda_{\mathbf{x}_{k} \rightarrow g_{\mathbf{x}}}\left(\mathbf{x}_{k}\right) \mathrm{d} \sim\left\{w_{k-1}\right\} .
\end{aligned}
$$

3 Now, $p\left(v_{k-1} \mid \mathbf{Z}_{1: k}\right)$ and $p\left(w_{k-1} \mid \mathbf{Z}_{1: k}\right)$ are computed by multiplying prior and incoming messages from linked likelihoods, and are respectively sent to $g_{v_{k}}$ and $g_{w_{k}}$.

4 Each variable receives the message from linked likelihoods, finally, $p\left(v_{k} \mid \mathbf{Z}_{1: k}\right)$ and $p\left(w_{k} \mid \mathbf{Z}_{1: k}\right)$ are determined. For instance, $p\left(w_{k} \mid \mathbf{Z}_{1: k}\right)$ is given by $p\left(w_{k} \mid \mathbf{Z}_{1: k}\right)=\int p\left(w_{k-1} \mid \mathbf{Z}_{1: k}\right) p\left(w_{k} \mid w_{k-1}\right) \mathrm{d} w_{k-1}$.

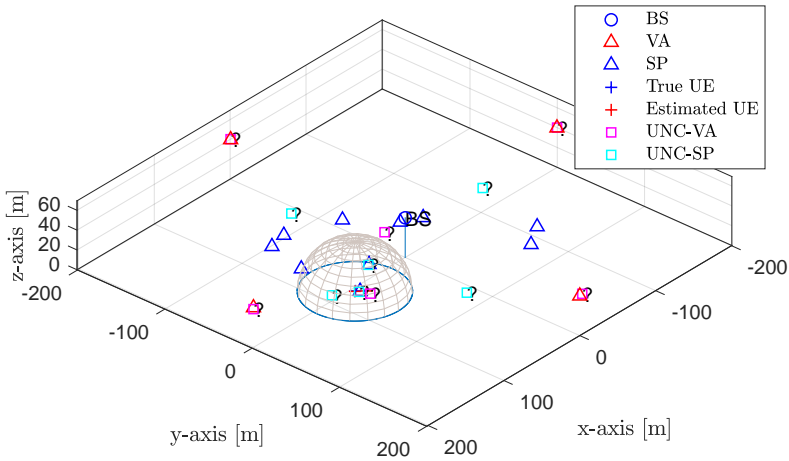

(a) $k=2$

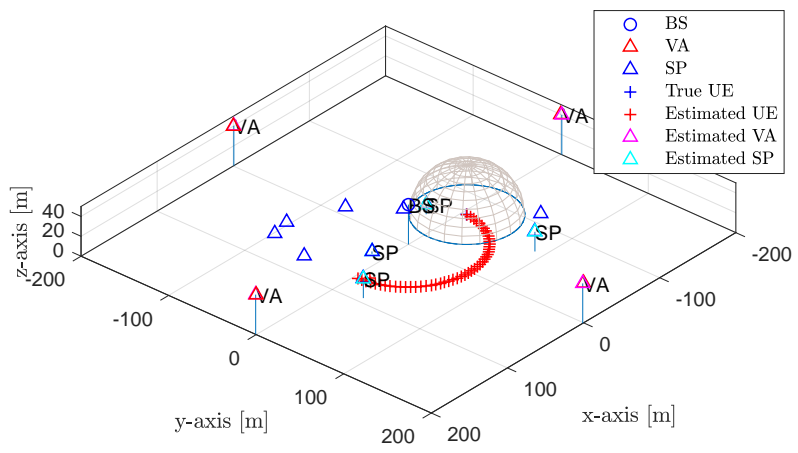

(b) $k=50$

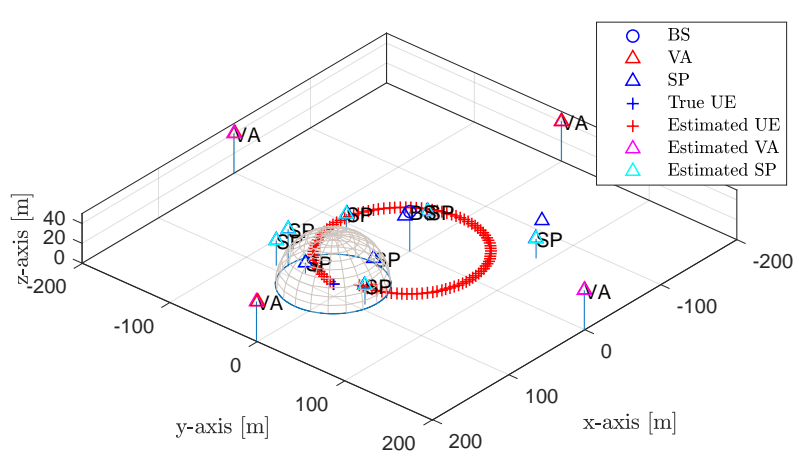

(c) $k=100$

Fig. 3. Exemplary result of map maintenance with the estimated UE, VAs, and SPs over time. The dome represents the visible SPs around the UE. UNC refers to an unconfirmed source.

\section{Simulations and Results}

\section{A. Simulation Setup}

We consider an environment where a UE is moving on a circular road with a base station, four VAs, and 10 randomly distributed SPs as shown in Fig. 3. The base station and four VAs are located at $[0,0,40]^{\mathrm{T}} \mathrm{m},[200,0,40]^{\mathrm{T}} \mathrm{m}$, $[0,200,40]^{\mathrm{T}} \mathrm{m},[-200,0,40]^{\mathrm{T}} \mathrm{m}$, and $[0,-200,40]^{\mathrm{T}} \mathrm{m}$ respectively. The SPs are visible only when the distance to the UE is less than 50 meters. The mobility of the UE follows the state model in Section II-A. The UE state is initialized as $\mathbf{x}_{1}=[70.73,0,0, \pi / 2,22.22, \pi / 10,300]^{\mathrm{T}}$, with units $\mathrm{m}, \mathrm{m}$, $\mathrm{m}, \mathrm{rad}, \mathrm{m} / \mathrm{s}, \mathrm{rad} / \mathrm{s}$, and $\mathrm{m}$. The epoch interval is $200 \mathrm{~ms}$. The process noise standard deviations are set to $\sigma_{v}=0.1 \mathrm{~m} / \mathrm{s}$, $\sigma_{\omega}=0.001 \mathrm{rad} / \mathrm{s}$, and $\sigma_{B}=0.1 \mathrm{~m}$. We have set $P_{\mathrm{D}}=1$ 

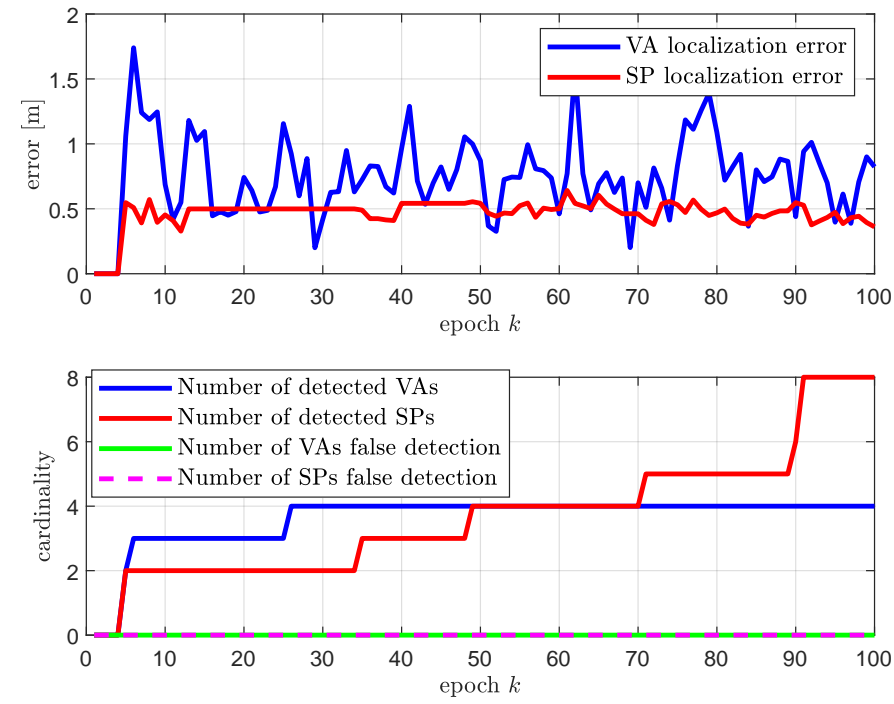

Fig. 4. Mapping performance in terms of localization error of confirmed sources (top) and number of confirmed sources (bottom).
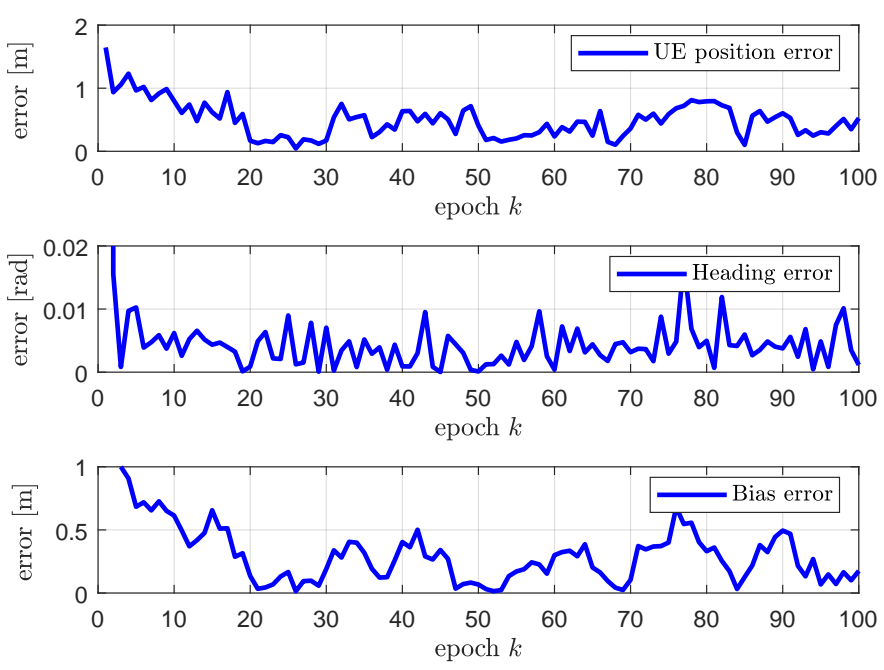

Fig. 5. Tracking performance of the UE location, heading, and clock bias.

and $P_{\mathrm{N}} \ll 1$. Initial prior distribution of the UE location is set to Gaussian with standard deviation of $14.2 \mathrm{~m}$ in the horizontal plane, but with the perfect knowledge of the vertical coordinate. The orientation, longitudinal velocity, rotational velocity, and clock bias priors are set to Gaussian with standard deviation of $1 \mathrm{rad}, 0.1 \mathrm{~m} / \mathrm{s}, 0.1 \mathrm{rad} / \mathrm{s}$, and $10 \mathrm{~m}$, respectively. The measurement covariance matrix $\Sigma_{l, k}$ is set to be diagonal, with $0.1 \mathrm{~m}$ standard deviation for TOA, $0.01 \mathrm{rad}$ standard deviation for both DOD and DOA. 2000 samples are utilized for approximating the posterior distributions, 10 iterations of message passing are performed at each epoch.

\section{B. Results and Discussion}

We evaluate the capability of the proposed algorithm to perform vehicle localization, vehicle heading and clock bias determination, and mapping. Fig. 3 shows an exemplary result of map maintenance which represents the estimated locations
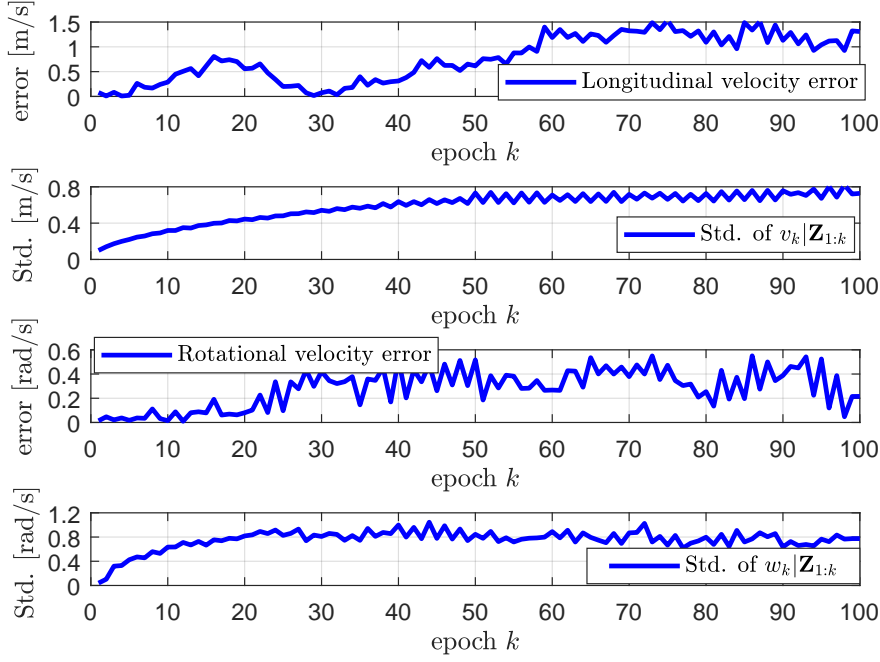

Fig. 6. Smoothing performance of the longitudinal and rotational velocity.

of UE, VAs, and SPs over time. At epoch $k=2$, the UE receives signals via 6 paths, each corresponding to BS and unconfirmed sources (4 VAs and $1 \mathrm{SP}$ ). As the UE moves along the circular road, SP sources within the visible distance to the UE, and VA sources can be found are eventually confirmed. After 100 epochs, the vehicle turns around the circular road, then the map comprises all existing sources. Fig. 4 shows the mapping performance. All unconfirmed sources are detected correctly. In case of the VA, two of four VAs are initially detected (confirmed) at $k=5$, and one more VA is detected at $k=6$, all VAs are confirmed at $k=26$. In case of the SP, two SPs are first confirmed at $k=5$, and the rest SPs are confirmed by $k=91$. The average localization error of the VA and SP over time is respectively $0.8 \mathrm{~m}$ and $0.6 \mathrm{~m}$. The localization error for the VA is larger than that of the SP due to the geometric configuration: the VAs are further away from the UE than the SPs. Fig. 5 shows the tracking performance for the UE location, heading, and clock bias. The average tracking errors of them over time are $0.5 \mathrm{~m}, 0.02 \mathrm{rad}$, and 0.3 $\mathrm{m}$, respectively. As compared to the results in [9], we were able to achieve decreasing errors over the course of tracking which justifies the efficacy of the proposed tracking method. In our vehicular tracking system, the belief of the longitudinal and rotational velocity can not be directly determined, but is rather obtained via the 1-step smoother. Fig. 6 shows the smoothing performance for the longitudinal and rotational velocity. Both velocity errors tend to increase over time, but remain less than $1.5 \mathrm{~m} / \mathrm{s}$ and $0.6 \mathrm{rad} / \mathrm{s}$ with the help of the 1-step smoother. Note that as shown in Fig. 2 the belief of the UE location and heading constrains the standard deviation of both velocities to be not diverging.

\section{CONCLUSION}

5G mmWave signal provides a tremendous possibility for vehicular tracking. We proposed a Bayesian $5 \mathrm{G}$ mmWave tracking filter consisting of the message passing and data association to solve a SLAM problem. We confirmed that the 
clock bias as well as vehicle heading/position are successfully estimated for a moving vehicle without any prior information. At the same time, the localization and mapping of VAs and SPs are also carried out seamlessly. It is expected that a practical implementation of the proposed algorithm will pave a way for autonomous driving with $5 \mathrm{G}$ mmWave signals.

\section{ACKNOWLEDGMENTS}

This work was supported, in part, by the EU H2020 projects HIGHTS MG-3.5a-2014-636537 and 5GCAR, and the VINNOVA COPPLAR project, funded under Strategic Vehicle Research and Innovation Grant No. 2015-04849, the Ministry of Science, ICT and Future Planning, Korea, under the ITRC support program (IITP-2018-0-01637) supervised by the Institute for Information \& communications Technology Promotion, Samsung Research Funding \& Incubation Center of Samsung Electronics under Project Number SRFC-IT-160109, the Spanish Ministry of Science, Innovation and Universities, under Grant TEC2017-89925-R.

\section{ApPENDiX: Relation BetWeEn ChanNel AND LOCATION PARAMETERS}

In this appendix, we detail the relation between the location parameters and the channel parameters. For conciseness of the notation, we drop the time index $k$ and the VA/SP index $m$.

LOS path: For the LOS path between BS and UE, we have the following relations: Delay: $\tau=\left\|\mathbf{x}_{\mathrm{BS}}-\mathbf{x}_{k}\right\| / c+B$, where $c$ denotes the speed of light. Direction of departure: $\phi_{0}^{\mathrm{az}}=$ $\arctan \left(y_{k} / x_{k}\right), \quad \phi_{0}^{\mathrm{el}}=\arcsin \left(\left(z_{k}-z_{\mathrm{BS}}\right) /\left(\left\|\mathbf{x}_{k}-\mathbf{x}_{\mathrm{BS}}\right\|\right)\right)$, where we assume $\arctan 2$ is used. Direction of arrival: We remind that the DOA is measured in the local frame of reference of the UE, so that the UE orientation must be accounted for: $\theta_{0}^{\mathrm{az}}=\pi+\arctan \left(y_{k} / x_{k}\right)-\alpha_{k}, \theta_{0}^{\mathrm{el}}=$ $\arcsin \left(\left(z_{\mathrm{BS}}-z_{k}\right) /\left(\left\|\mathbf{x}_{\mathrm{BS}}-\mathbf{x}_{k}\right\|\right)\right)$, since the DOA elevation measurement does not depend on the UE orientation.

Virtual Anchors: Between a virtual anchor $\mathbf{x}_{\mathrm{VA}}$ and the user's position $\mathbf{x}_{k}$, the incidence point of the specular reflection on the reflecting surface is given by the point where the straight line between the VA and UE crosses the reflecting surface, as shown in Fig. 1. It is given by

$$
\mathbf{x}_{s}=\mathbf{x}_{\mathrm{VA}}+\frac{\left(\mathbf{f}-\mathbf{x}_{\mathrm{VA}}\right)^{\mathrm{T}} \mathbf{u}}{\left(\mathbf{x}_{k}-\mathbf{x}_{\mathrm{VA}}\right)^{\mathrm{T}} \mathbf{u}}\left(\mathbf{x}_{k}-\mathbf{x}_{\mathrm{VA}}\right) \text {. }
$$

Here, $\mathbf{u}=\left(\mathbf{x}_{\mathrm{BS}}-\mathbf{x}_{\mathrm{VA}}\right) /\left\|\left(\mathbf{x}_{\mathrm{BS}}-\mathbf{x}_{\mathrm{VA}}\right)\right\|$ and $\mathbf{f}=\left(\mathbf{x}_{\mathrm{BS}}+\mathbf{x}_{\mathrm{VA}}\right) / 2$. Note that this allows to find explicit expressions of $\mathbf{x}_{s}$ that only depend on $\mathbf{x}_{\mathrm{VA}}, \mathbf{x}_{\mathrm{BS}}$, and $\mathbf{x}_{k}$ (not shown). Conversely, the location of a VA can be expressed as a function of the incidence point:

$$
\mathbf{x}_{\mathrm{VA}}=\mathbf{x}_{k}+\left(\left\|\mathbf{x}_{k}-\mathbf{x}_{s}\right\|+\left\|\mathbf{x}_{\mathrm{BS}}-\mathbf{x}_{s}\right\|\right) \frac{\mathbf{x}_{s}-\mathbf{x}_{k}}{\left\|\mathbf{x}_{s}-\mathbf{x}_{k}\right\|} .
$$

Next, we state the relations between the channel parameters $\tau, \boldsymbol{\theta}=\left[\theta^{\mathrm{el}}, \theta^{\mathrm{az}}\right]^{\mathrm{T}}$, and $\boldsymbol{\phi}=\left[\phi^{\mathrm{el}}, \phi^{\mathrm{az}}\right]^{\mathrm{T}}$ and the system state. Delays: $\tau=\left\|\mathbf{x}_{\mathrm{VA}}-\mathbf{x}_{k}\right\| / c+B$. This is equivalent to $\tau=$ $\left\|\mathbf{x}_{\mathrm{BS}}-\mathbf{x}_{s}\right\| / c+\left\|\mathbf{x}_{s}-\mathbf{x}_{k}\right\| / c+B$. Direction of departure: $\phi^{\mathrm{az}}=$ $\arctan \left(y_{\mathrm{s}} / x_{\mathrm{s}}\right)$ and $\phi^{\mathrm{el}}=\arcsin \left(\left(z_{\mathrm{s}}-z_{\mathrm{BS}}\right) /\left(\left\|\mathbf{x}_{\mathrm{s}}-\mathbf{x}_{\mathrm{BS}}\right\|\right)\right)$. Direction of arrival: $\theta^{\mathrm{az}}=\arctan \left(\left(y_{\mathrm{VA}}-y_{k}\right) /\left(x_{\mathrm{VA}}-x_{k}\right)\right)-$ $\alpha_{k}$ and $\theta^{\mathrm{el}}=\arcsin \left(\left(z_{\mathrm{VA}}-z_{k}\right) /\left(\left\|\mathbf{x}_{\mathrm{VA}}-\mathbf{x}_{k}\right\|\right)\right)$.
Scattering Points: For SPs, the relations are largely a special case of the VAs. We here only note the differences, considering an SP with location $\mathbf{x}_{\mathrm{SP}}$. Delays: $\tau=\left\|\mathbf{x}_{\mathrm{SP}}-\mathbf{x}_{\mathrm{BS}}\right\| / c+\| \mathbf{x}_{\mathrm{SP}}-$ $\mathbf{x}_{k} \| / c+B$. Direction of departure: $\phi^{\mathrm{az}}=\arctan \left(y_{\mathrm{SP}} / x_{\mathrm{SP}}\right)$ and $\phi^{\mathrm{el}}=\arcsin \left(\left(z_{\mathrm{SP}}-z_{\mathrm{BS}}\right) /\left(\left\|\mathbf{x}_{\mathrm{SP}}-\mathbf{x}_{\mathrm{BS}}\right\|\right)\right)$. Direction of arrival: $\theta^{\mathrm{az}}=\arctan \left(\left(y_{\mathrm{SP}}-y_{k}\right) /\left(x_{\mathrm{SP}}-x_{k}\right)\right)-\alpha_{k}$ and $\theta^{\mathrm{el}}=$ $\arcsin \left(\left(z_{\mathrm{SP}}-z_{k}\right) /\left(\left\|\mathbf{x}_{\mathrm{SP}}-\mathbf{x}_{k}\right\|\right)\right)$.

\section{REFERENCES}

[1] P. D. Groves and Z. Jiang, "Height aiding, $C / N_{0}$ weighting and consistency checking for GNSS NLOS and multipath mitigation in urban areas," J. Navigat., vol. 66, no. 5, pp. 653-669, Sep. 2013.

[2] S. M. Patole, M. Torlak, D. Wang, and M. Ali, "Automotive radars: A review of signal processing techniques," IEEE Signal Process. Mag., vol. 34, no. 2, pp. 22-35, Mar. 2017.

[3] H. Wymeersch, G. Seco-Granados, G. Destino, D. Dardari, and F. Tufvesson, " $5 \mathrm{G} \mathrm{mm}$-Wave positioning for vehicular networks," Wireless Commun. Mag., vol. 24, no. 6, pp. 80-86, Dec. 2018.

[4] K. Witrisal, P. Meissner, E. Leitinger, Y. Shen, C. Gustafson, F. Tufvesson, K. Haneda, D. Dardari, A. F. Molisch, A. Conti et al., "Highaccuracy localization for assisted living: $5 \mathrm{G}$ systems will turn multipath channels from foe to friend," IEEE Signal Processing Magazine, vol. 33, no. 2, pp. 59-70, 2016.

[5] H. Durrant-Whyte and T. Bailey, "Simultaneous localization and mapping: part I," IEEE Robot. Autom. Mag., vol. 13, no. 2, pp. 99-110, Jun. 2006.

[6] F. Meyer, T. Kropfreiter, J. L. Williams, R. Lau, F. Hlawatsch, P. Braca, and M. Z. Win, "Message passing algorithms for scalable multitarget tracking," Proceedings of the IEEE, vol. 106, no. 2, pp. 221-259, 2018.

[7] F. Guidi, A. Guerra, and D. Dardari, "Personal mobile radars with millimeter-wave massive arrays for indoor mapping," IEEE Trans. Mobile Comput., vol. 15, no. 6, pp. 1471-1484, Jun. 2016.

[8] A. Shahmansoori, G. E. Garcia, G. Destino, G. Seco-Granados, and $\mathrm{H}$. Wymeersch, "Position and orientation estimation through millimeterwave MIMO in 5G systems," IEEE Trans. Wireless Commun., vol. 17, no. 3, pp. 1822-1835, Mar. 2018.

[9] H. Wymeersch, N. Garia, H. Kim, G. Seco-Granados, S. Kim, F. Wen, and M. Fröhle, "5G mmwave downlink vehicular positioning," in IEEE Globecom, Dec. 2018.

[10] S. Thrun, W. Burgard, and D. Fox, "Probabilistic robotics (intelligent robotics and autonomous agents series). intelligent robotics and autonomous agents," The MIT Press, vol. 2, p. 47, 2005.

[11] R. Schubert, E. Richter, and G. Wanielik, "Comparison and evaluation of advanced motion models for vehicle tracking," in Information Fusion, 2008 11th International Conference on. IEEE, 2008, pp. 1-6.

[12] R. W. Heath, N. González-Prelcic, S. Rangan, W. Roh, and A. M. Sayeed, "An overview of signal processing techniques for millimeter wave MIMO systems," IEEE J. Sel. Topics Signal Process., vol. 10, no. 3, pp. 436-453, Apr. 2016.

[13] F. Roemer, M. Haardt, and G. D. Galdo, "Analytical performance assessment of multi-dimensional matrix- and tensor-based ESPRIT-type algorithms," IEEE Trans. Signal Process., vol. 62, no. 10, pp. 26112625, May 2014.

[14] Y. Bar-Shalom and X.-R. Li, Multitarget-multisensor tracking: principles and techniques. YBS, UK, 1995.

[15] J. Munkres, "Algorithms for the assignment and transportation problems," J. Soc. Indust. Appl. Math., vol. 5, no. 1, pp. 32-38, Mar. 1957.

[16] F. Bourgeois and J.-C. Lassalle, "An extension of the munkres algorithm for the assignment problem to rectangular matrices," Commun. ACM, vol. 14, no. 12, pp. 802-804, Dec. 1971.

[17] Z. Abu-Shaban, X. Zhou, T. Abhayapala, G. Seco-Granados, and H. Wymeersch, "Error bounds for uplink and downlink 3D localization in 5G mmwave systems," IEEE Transactions on Wireless Communications, vol. 17, no. 8, pp. 4939-4954, Aug 2018. 